\title{
Shape analysis in Higgs boson pair production
}

\section{Matteo Capozi, Gudrun Heinrich*}

Max Planck Institute for Physics, Föhringer Ring 6, 80805 Munich, Germany

E-mail: mcapozi@mpp.mpg •de, gudrun@mpp.mpg · de

We study the impact of anomalous couplings in the Higgs sector on the shape of the Higgs boson pair invariant mass distribution at NLO. Our analysis is based on a five-dimensional coupling parameter space relevant for Higgs boson pair production in gluon fusion, in the framework of a non-linear Effective Field Theory. In particular, we present a clustering procedure into certain shape types based on unsupervised machine learning, with the aim to infer information about the underlying parameter space from a given shape type.

14th International Symposium on Radiative Corrections (RADCOR2019)

9-13 September 2019

Palais des Papes, Avignon, France

${ }^{*}$ Speaker. 


\section{Introduction}

Higgs boson pair production in gluon fusion is an important process to extract information about the trilinear Higgs boson coupling $\lambda$. Recent LHC measurements $[1,2]$ have used this process to constrain $\lambda$ to the range $-5.0 \leq \lambda / \lambda_{\mathrm{SM}} \leq 12.0$ [1]. Combined constraints, based on indirect measurements from single Higgs production processes and direct limits from double Higgs boson production lead to $-2.3 \leq \lambda / \lambda_{\mathrm{SM}} \leq 10.3$ [3]. However the latter constraints strongly depend on the assumption that all deviations from the SM expectation are stemming from a modification of the trilinear coupling only, while the other couplings are fixed to their SM values.

On the theory side, various attempts have been made to come up with constraints on $c_{h h h}=$ $\lambda / \lambda_{\mathrm{SM}}$ that are largely model independent [4-7], suggesting $\left|c_{h h h}\right| \lesssim 4$ for a new physics scale in the few $\mathrm{TeV}$ range. Recent phenomenological studies about the precision that could be reached for the self-couplings at the (HL-)LHC and future hadron colliders are summarised in Refs. [8-11].

Higgs boson pair production in gluon fusion in the SM has been calculated at leading order in Refs. [12-14], and at NLO in Ref. [15] in the $m_{t} \rightarrow \infty$ limit ("heavy top limit, HTL"), rescaled with the full Born matrix element. The NLO QCD corrections with full top quark mass dependence have been calculated in Refs. [16-18]. Implementations of the full NLO QCD corrections in parton shower Monte Carlo programs are also available [19-21]. The uncertainties due to the chosen top mass scheme have been assessed in Ref. [18].

In the HTL, the NNLO QCD corrections have been computed in Refs. [22-26], the $\mathrm{N}^{3} \mathrm{LO}$ corrections in Ref. [27]. The calculation of Ref. [26] has been combined with results including the top quark mass dependence as far as available in Ref. [28], and the latter has been supplemented by soft gluon resummation in Ref. [29]. Analytic approximations to the top-mass dependent two-loop amplitudes in several limits, as well as phase space integrals for the NNLO real radiation, have been studied in Refs. [30-34]. Complete analytic results in the high energy limit have been presented in Ref. [35]; the latter have been combined with the full NLO results, in the regions where they are more appropriate, in Ref. [36].

The effects of operators within an Effective Field Theory (EFT) description of Higgs boson pair production have been studied at NLO in the HTL in Refs. [37-40]. EFT studies at NNLO in the HTL are also available [41]. In Ref. [42] for the first time the full NLO QCD corrections have been combined with an EFT approach to study BSM effects.

It is well known that Higgs boson pair production in gluon fusion is a process where delicate cancellations occur between triangle-type contributions, which contain the trilinear Higgs coupling, and box-type contributions. A deviation of the trilinear coupling from its SM value would change this interference pattern and thus lead to characteristic shape changes in the di-Higgs invariant mass distribution $m_{h h}$. However, the question arises whether such shape changes could as well be induced by a combination of other anomalous couplings in the Higgs sector, thus faking an anomalous value for $\lambda$. Therefore, in Ref. [43], we performed a shape analysis based on a 5-dimensional coupling parameter space and proposed a method to associate certain shapes with distinct regions in the parameter space.

The idea of a shape analysis has been pursued already in various ways based on LO studies, see e.g. Refs. [44-49]. In Ref. [47], a cluster analysis has been proposed to define 12 benchmark points 
in a 5-dimensional non-linear EFT parameter space which result from clusters of "similar" shapes. The similarity measure in this case is based on a binned likelihood ratio using LO predictions for the observables $m_{h h}, \cos \theta^{*}$ and $p_{T, h}$. In Ref. [42] it was analysed how the $m_{h h}$ and $p_{T, h}$ distributions change when going from LO to NLO for the benchmark points defined in Ref. [47], and it was found that the NLO corrections can have a significant impact on the shapes.

We do not attempt to define new benchmark points here, but rather would like to identify more global patterns in the $m_{h h}$ spectrum which can be attributed to coupling configurations. To this aim we apply an unsupervised learning algorithm to identify patterns in the shapes of the $m_{h h}$ distribution. Then we use the KMeans clustering algorithm from scikit-learn [50] and ask for a classification of the shapes into a given number of clusters, and finally relate the clusters to the underlying parameter space.

\section{Parametrisation of anomalous couplings in the Higgs sector}

Our studies are based on an effective Lagrangian in a non-linear Effective Field Theory ("Higgs Effective Field Theory, HEFT") [42,51] relevant for Higgs boson pair production, assuming CP conservation:

$$
\mathscr{L} \supset-m_{t}\left(c_{t} \frac{h}{v}+c_{t t} \frac{h^{2}}{v^{2}}\right) \bar{t} t-c_{h h h} \frac{m_{h}^{2}}{2 v} h^{3}+\frac{\alpha_{s}}{8 \pi}\left(c_{g g h} \frac{h}{v}+c_{g g h h} \frac{h^{2}}{v^{2}}\right) G_{\mu v}^{a} G^{a, \mu v} .
$$

In the SM $c_{t}=c_{h h h}=1$ and $c_{t t}=c_{g g h}=c_{g g h h}=0$. The chromomagnetic operator is absent in (2.1) because it contributes to $g g \rightarrow h h$ only at higher order in the counting underlying the HEFT Lagrangian [42]. The coefficients $c_{g g h}$ and $c_{g g h h}$ are related in SMEFT ("SM Effective Field Theory") [11], however in HEFT they are a priori independent.

We produce our data using the differential distributions calculated in Ref. [42], parametrised in terms of 23 coefficients $A_{i}$ for each coupling combination occurring in the (differential) NLO cross section, which allows for a fast evaluation:

$$
\begin{aligned}
\frac{d \sigma}{d m_{h h}}= & A_{1} c_{t}^{4}+A_{2} c_{t t}^{2}+A_{3} c_{t}^{2} c_{h h h}^{2}+A_{4} c_{g h h}^{2} c_{h h h}^{2}+A_{5} c_{g g h}^{2}+A_{6} c_{t t} c_{t}^{2}+A_{7} c_{t}^{3} c_{h h h} \\
& +A_{8} c_{t t} c_{t} c_{h h h}+A_{9} c_{t t} c_{g g h} c_{h h h}+A_{10} c_{t t} c_{c g g h}+A_{11} c_{t}^{2} c_{g g h} c_{h h h}+A_{12} c_{t}^{2} c_{g g h h} \\
& +A_{13} c_{t} c_{h h h}^{2} c_{g h h}+A_{14} c_{t} c_{h h h} c_{g g h h}+A_{15} c_{g g h} c_{h h h} c_{g g h h}+A_{16} c_{t}^{3} c_{g g h}+A_{17} c_{t} c_{t t} c_{g g h} \\
& +A_{18} c_{t} c_{g g h}^{2} c_{h h h}+A_{19} c_{t} c_{g g h} c_{g g h h}+A_{20} c_{t}^{2} c_{g g h}^{2}+A_{21} c_{t t} c_{g g h}^{2}+A_{22} c_{g g h}^{3} c_{h h h} \\
& +A_{23} c_{g g h}^{2} c_{g g h h} .
\end{aligned}
$$

The coefficients $A_{i}$ are available in bins of width $20 \mathrm{GeV}$ from $250 \mathrm{GeV}$ to $1050 \mathrm{GeV}$.

\section{Shape classification and clustering}

There is no need to use machine learning for shape classifications, it is certainly possible to write an analyser function that checks the slopes of the distributions and puts them into predefined shape classes. However it turned out that this procedure is cumbersome, as it should take into account bin-by-bin variations as well as more global features of the $m_{h h}$ spectrum, and it is hard to 
extend to a larger number of shape types. Therefore, to avoid the bias introduced by the "manual" definition of the shape types, and to find a more flexible classification which can be extended easily to more shape types, we devised a different approach to the classification problem, using unsupervised learning techniques.

We constructed a classification of the shapes of the $m_{h h}$ distribution into a given number of shape clusters, where we did not predefine what the clusters should look like. For this purpose we used an autoencoder to find common patterns in the data. The setup is implemented using Keras [52] and TensorFlow [53]. As input data we used 30 bins of width $20 \mathrm{GeV}$ for the normalised $m_{h h}$ distributions. We trained a neural network based on a set of $10^{5}$ distributions, produced by variations of the coupling parameters in ranges as specified below.

To reduce uncertainties, for example due to overfitting, we produced ten different autoencoder models, where for each model we picked $10^{4}$ random points from the training set for validation. The ten models all have the same goal, but are starting from different training and validation sets and thus a different initialisation of the weights. We trained the autoencoder for each model over 10000 epochs using Adam [54] as optimiser and the root mean square error to define the loss function. The ten different encoded training data sets are then fed to a classification algorithm, where we employed the KMeans clustering algorithm from scikit-learn [50], asking for a classification into a given number of clusters, where we tested classifications into 4-8 clusters. Asking the KMeans algorithm to find 4 and 7 clusters yielded the shape types shown in Fig. 1. The curves denote the cluster centres determined by the KMeans algorithm, for each of the ten encoder models.

One can see from Fig. 1 that in the case of clustering into four shape types, cluster 2 contains shapes which vary substantially. In contrast, for seven shape types, the cluster centres obtained from the ten different encoder models are quite similar. Applying the procedure to 4-8 clusters revealed that seven clusters seemed to be the optimal number to capture distinct shape features, while defining more than seven clusters did not lead to useful additional features, but rather to the tendency to focus on local minima in the clustering space.

To combine the results from the ten autoencoder models, we adopted the "majority vote" method, i.e. for each of the ten outcomes, a given point in the coupling parameter space gets a label ("vote") corresponding to the cluster it belongs to. The final cluster assigned to that point is the one which collected the largest number of votes. 

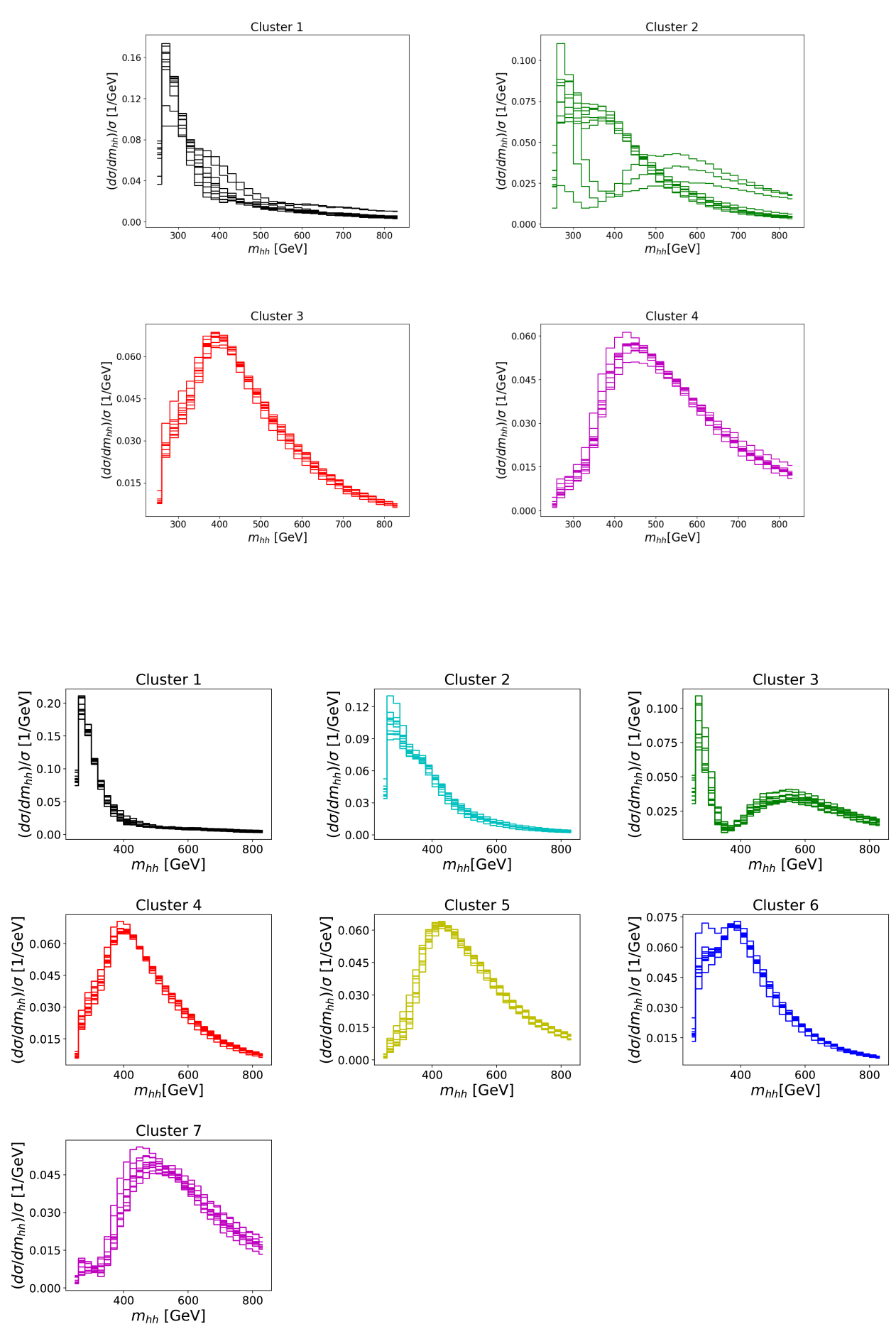

Figure 1: The clusters obtained by asking for a classification into four or seven shape types. The cluster centres obtained from 10 different encoder models are shown, in the colour codes used later to visualise the underlying parameter space. 


\section{Parameter space underlying the clusters}

In this section we show how the parameter space relates to the clusters if we ask for 4 or 7 clusters. Our results for the $g g \rightarrow h h$ cross sections at NLO are produced for a centre-of-mass energy of $\sqrt{s}=13$ TeV, using PDF4LHC15_nlo_100_pdfas [55] parton distribution functions interfaced via LHAPDF, along with the corresponding value for $\alpha_{s}$. The masses have been set to $m_{h}=125 \mathrm{GeV}$, $m_{t}=173 \mathrm{GeV}$ and the top quark width has been set to zero. We study the differential cross section as a function of five anomalous couplings, varying them in the ranges specified below:

$$
c_{t} \in[0.5,1.5], c_{h h h} \in[-3,8], c_{t t} \in[-3,3], c_{g g h}, c_{g g h h} \in[-0.5,0.5] .
$$

The ranges are motivated by current experimental constraints. For $c_{h h h}$ we use a smaller range than constrained by experiment in order to focus more on the range where interesting shape changes occur. In order to visualise the results, we project out 2-dimensional slices of the 5-dimensional parameter space, fixing the other three couplings to their SM values. The colour codes are shown

\begin{tabular}{|c|c|c|}
\hline Cluster & type & colour \\
\hline \hline \multicolumn{3}{|c|}{ 4 clusters } \\
\hline \hline 1 & enhanced low $m_{h h}$ & black \\
3 & double peak/shoulder & green \\
4 & SM-like & red \\
magenta
\end{tabular}

Table 1: Clusters and shape types with corresponding colour codes for the classification into four and seven clusters.

in Fig. 1, and are also listed in Table 1.

In Fig. 2 we display shape types resulting from variations of $c_{t}$ versus $c_{h h h}$. We observe that the clustering into seven clusters provides a much more refined distinction of SM-like shapes from distributions with an enhanced tail or a doubly peaked structure than the classification into four clusters. The figure clearly shows that small variations of $c_{h h h}$ can easily distort the SM-like shape, while the shape is more robust against variations of $c_{t}$. Fig. 3 shows $c_{t t}$ versus $c_{h h h}$, where we see that the interplay between $c_{h h h}$ and $c_{t t}$ can lead to all shape types. Small deviations of these couplings from the SM value already can have a substantial effect on the shape. Furthermore, it becomes apparent that $c_{t t}$ values different from zero enhance the total cross section, such that limits on the total cross section combined with shape information allows to constrain $c_{t t}$. 

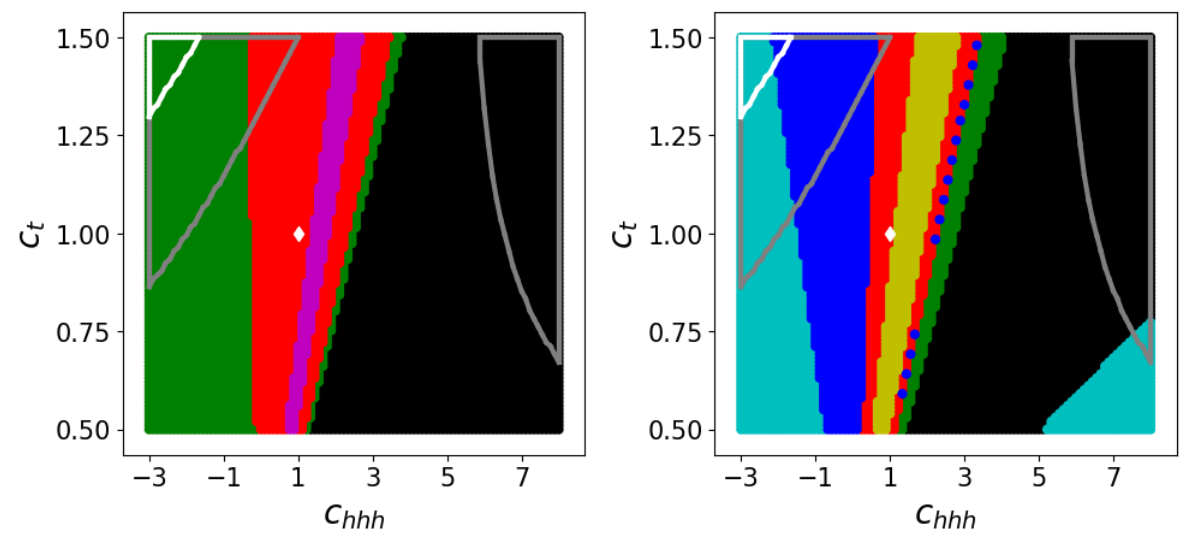

Figure 2: Shape types produced by variations of $c_{t}$ versus $c_{h h h}$. Left: 4 clusters, right: 7 clusters. The areas outside the silver and white curves are regions where the total cross section exceeds $6.9 \times \sigma_{S M}$ and $22.2 \times$ $\sigma_{S M}$, respectively. These values are motivated by the current ATLAS/CMS limits at $\sqrt{s}=13 \mathrm{TeV}$ [1,2]. The white diamond denotes SM parameter point. The colour code is given in Table 1.
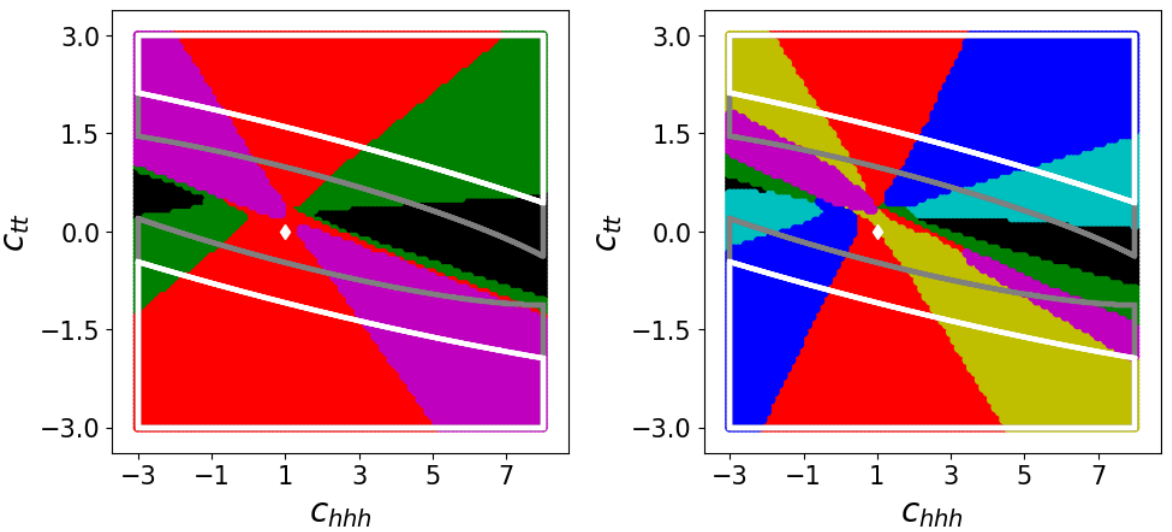

Figure 3: Shape types produced by variations of $c_{t t}$ versus $c_{h h h}$. Left: 4 clusters, right: 7 clusters. The areas outside the silver and white curves are regions where the total cross section exceeds $6.9 \times \sigma_{S M}$ and $22.2 \times \sigma_{S M}$, respectively.

Projections of all coupling parameter combinations onto two-dimensional planes show [43] that the parameters $c_{h h h}$ and $c_{t t}$ have the largest influence on the shape. In SMEFT, $c_{t t}$ is suppressed compared to $c_{t}$ by one order of the large new physics scale [11]. Furthermore, SMEFT imposes the relation $c_{g g h}=2 c_{g g h h}$. Using this relation and imposing that $c_{t t}$ amounts to $5 \%$ of $c_{t}$, we obtain a 3-dimensional parameter space simulating the SMEFT situation, which is visualised in Fig. 4.

\section{Conclusions}

We have identified shape clusters for the Higgs boson pair invariant mass distribution $m_{h h}$ based on an unsupervised machine learning approach. Using these clusters we investigated how anomalous couplings in the Higgs sector affect the shape of the $m_{h h}$ distribution. We found that the trilinear coupling $c_{h h h}$ and an effective $t \bar{t} h h$ coupling, $c_{t t}$, have the largest influence on the 


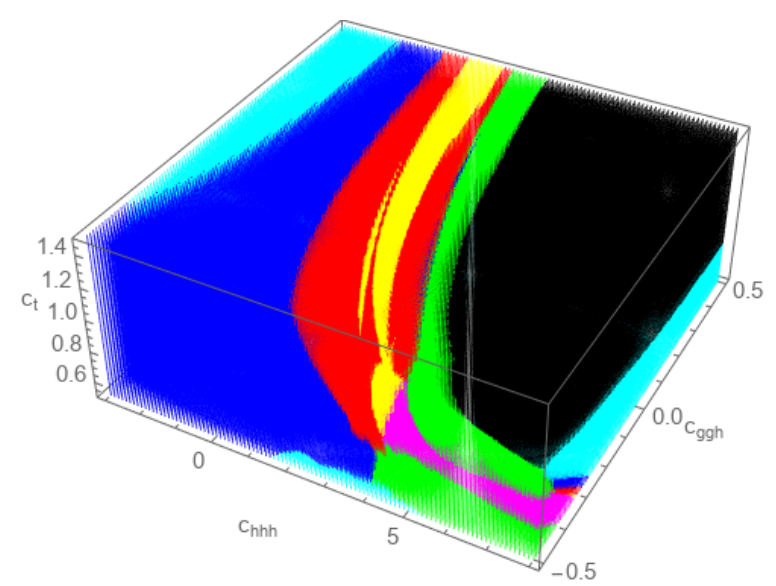

Figure 4: Three-dimensional visualisation of shape types produced by variations of $c_{t}, c_{h h h}$ and $c_{g g h}$. For the parameters not shown we used $c_{g g h h}=0.5 c_{g g h}$ and $c_{t t}=0.05 c_{t}$, simulating the SMEFT situation.

shape, while variations of the top Yukawa coupling and effective gluon-Higgs couplings in the experimentally allowed ranges have a smaller shape-changing effect. As the SM-like shape is not very robust against variations of $c_{h h h}$, combined information about the shape of the $m_{h h}$ spectrum and the total cross section has some potential to reveal New Physics effects at the High-LuminosityLHC.

\section{References}

[1] ATLAS collaboration, G. Aad et al., Combination of searches for Higgs boson pairs in pp collisions at $\sqrt{s}=13$ TeV with the ATLAS detector, Phys. Lett. B800 (2020) 135103 [1 906.02025 ].

[2] CMS collaboration, A. M. Sirunyan et al., Combination of searches for Higgs boson pair production in proton-proton collisions at $\sqrt{s}=13 \mathrm{TeV}$, Phys. Rev. Lett. 122 (2019) 121803 [1811. 09689 ].

[3] ATLAS collaboration, Constraints on the Higgs boson self-coupling from the combination of single-Higgs and double-Higgs production analyses performed with the ATLAS experiment, Tech. Rep. ATL-PHYS-PUB-2019-049, CERN, Geneva, Oct, 2019.

[4] A. Falkowski and R. Rattazzi, Which EFT, JHEP 10 (2019) 255 [1902 . 05936].

[5] S. Chang and M. A. Luty, The Higgs Trilinear Coupling and the Scale of New Physics, 1902.05556 .

[6] L. Di Luzio, R. Gröber and M. Spannowsky, Maxi-sizing the trilinear Higgs self-coupling: how large could it be?, Eur. Phys. J. C77 (2017) 788 [1704.02311].

[7] S. Di Vita, C. Grojean, G. Panico, M. Riembau and T. Vantalon, A global view on the Higgs self-coupling, JHEP 09 (2017) 069 [1704.01953].

[8] S. Dawson, C. Englert and T. Plehn, Higgs Physics: It ain't over till it's over, Phys. Rept. 816 (2019) $1[1808.01324]$.

[9] P. Basler, S. Dawson, C. Englert and M. Mühlleitner, Showcasing HH production: Benchmarks for the LHC and HL-LHC, Phys. Rev. D99 (2019) 055048 [1812.03542]. 
[10] M. Cepeda et al., Higgs Physics at the HL-LHC and HE-LHC, HL/HE WG2 group report, 1902.00134.

[11] J. Alison et al., Higgs Boson Pair Production at Colliders: Status and Perspectives, in Double Higgs Production at Colliders Batavia, IL, USA, September 4, 2018-9, 2019 (B. Di Micco, M. Gouzevitch, J. Mazzitelli and C. Vernieri, eds.), 2019, 1910.00012, https://lss.fnal.gov/archive/2019/conf/fermilab-conf-19-468-e-t.pdf.

[12] O. J. P. Eboli, G. C. Marques, S. F. Novaes and A. A. Natale, Twin Higgs Boson Production, Phys. Lett. B197 (1987) 269.

[13] E. W. N. Glover and J. J. van der Bij, Higgs Boson Pair Production via Gluon Fusion, Nucl. Phys. B309 (1988) 282.

[14] T. Plehn, M. Spira and P. M. Zerwas, Pair production of neutral Higgs particles in gluon-gluon collisions, Nucl. Phys. B479 (1996) 46 [hep-ph/ 9603205$].$

[15] S. Dawson, S. Dittmaier and M. Spira, Neutral Higgs boson pair production at hadron colliders: QCD corrections, Phys. Rev. D58 (1998) 115012 [hep-ph/9805244].

[16] S. Borowka, N. Greiner, G. Heinrich, S. P. Jones, M. Kerner, J. Schlenk et al., Higgs Boson Pair Production in Gluon Fusion at Next-to-Leading Order with Full Top-Quark Mass Dependence, Phys. Rev. Lett. 117 (2016) 012001 [1604.06447].

[17] S. Borowka, N. Greiner, G. Heinrich, S. P. Jones, M. Kerner, J. Schlenk et al., Full top quark mass dependence in Higgs boson pair production at NLO, JHEP 10 (2016) 107 [1608.04798].

[18] J. Baglio, F. Campanario, S. Glaus, M. Mühlleitner, M. Spira and J. Streicher, Gluon fusion into Higgs pairs at NLO QCD and the top mass scheme, Eur. Phys. J. C79 (2019) 459 [1811 . 05692 ].

[19] G. Heinrich, S. P. Jones, M. Kerner, G. Luisoni and E. Vryonidou, NLO predictions for Higgs boson pair production with full top quark mass dependence matched to parton showers, JHEP 08 (2017) 088 [1703.09252].

[20] S. Jones and S. Kuttimalai, Parton Shower and NLO-Matching uncertainties in Higgs Boson Pair Production, JHEP 02 (2018) 176 [1711.03319].

[21] G. Heinrich, S. P. Jones, M. Kerner, G. Luisoni and L. Scyboz, Probing the trilinear Higgs boson coupling in di-Higgs production at NLO QCD including parton shower effects, JHEP 06 (2019) 066 [1903.08137].

[22] D. de Florian and J. Mazzitelli, Two-loop virtual corrections to Higgs pair production, Phys. Lett. B724 (2013) 306 [1305.5206].

[23] D. de Florian and J. Mazzitelli, Higgs Boson Pair Production at Next-to-Next-to-Leading Order in QCD, Phys. Rev. Lett. 111 (2013) 201801 [1309.6594].

[24] J. Grigo, K. Melnikov and M. Steinhauser, Virtual corrections to Higgs boson pair production in the large top quark mass limit, Nucl. Phys. B888 (2014) 17 [1408. 2422].

[25] J. Grigo, J. Hoff and M. Steinhauser, Higgs boson pair production: top quark mass effects at NLO and NNLO, Nucl. Phys. B900 (2015) 412 [1508 . 00909 ].

[26] D. de Florian, M. Grazzini, C. Hanga, S. Kallweit, J. M. Lindert, P. Maierhöfer et al., Differential Higgs Boson Pair Production at Next-to-Next-to-Leading Order in QCD, JHEP 09 (2016) 151 [1606.09519]. 
[27] L.-B. Chen, H. T. Li, H.-S. Shao and J. Wang, Higgs boson pair production via gluon fusion at $N^{3} L O$ in $Q C D, 1909.06808$.

[28] M. Grazzini, G. Heinrich, S. Jones, S. Kallweit, M. Kerner, J. M. Lindert et al., Higgs boson pair production at NNLO with top quark mass effects, JHEP 05 (2018) 059 [1803. 02463 ].

[29] D. De Florian and J. Mazzitelli, Soft gluon resummation for Higgs boson pair production including finite $M_{t}$ effects, JHEP 08 (2018) 156 [1807 . 03704 ].

[30] R. Gröber, A. Maier and T. Rauh, Reconstruction of top-quark mass effects in Higgs pair production and other gluon-fusion processes, JHEP 03 (2018) 020 [1 709.07799 ].

[31] R. Bonciani, G. Degrassi, P. P. Giardino and R. Gröber, Analytical Method for Next-to-Leading-Order QCD Corrections to Double-Higgs Production, Phys. Rev. Lett. 121 (2018) 162003 [1806.11564].

[32] X. Xu and L. L. Yang, Towards a new approximation for pair-production and associated-production of the Higgs boson, JHEP 01 (2019) 211 [1810 . 12002].

[33] J. Davies, G. Mishima, M. Steinhauser and D. Wellmann, Double-Higgs boson production in the high-energy limit: planar master integrals, JHEP 03 (2018) 048 [1801. 09696 ].

[34] J. Davies, F. Herren, G. Mishima and M. Steinhauser, Real-virtual corrections to Higgs boson pair production at NNLO: three closed top quark loops, JHEP 05 (2019) 157 [1904.11998].

[35] J. Davies, G. Mishima, M. Steinhauser and D. Wellmann, Double Higgs boson production at NLO in the high-energy limit: complete analytic results, JHEP 01 (2019) 176 [1811. 05489 ].

[36] J. Davies, G. Heinrich, S. P. Jones, M. Kerner, G. Mishima, M. Steinhauser et al., Double Higgs boson production at NLO: combining the exact numerical result and high-energy expansion, JHEP 11 (2019) 024 [1907.06408].

[37] R. Gröber, M. Mühlleitner, M. Spira and J. Streicher, NLO QCD Corrections to Higgs Pair Production including Dimension-6 Operators, JHEP 09 (2015) 092 [1504 06577 ].

[38] R. Gröber, M. Mühlleitner and M. Spira, Signs of Composite Higgs Pair Production at Next-to-Leading Order, JHEP 06 (2016) 080 [1602 . 05851 ].

[39] F. Maltoni, E. Vryonidou and C. Zhang, Higgs production in association with a top-antitop pair in the Standard Model Effective Field Theory at NLO in QCD, JHEP 10 (2016) 123 [1607. 05330 ].

[40] R. Gröber, M. Mühlleitner and M. Spira, Higgs Pair Production at NLO QCD for CP-violating Higgs Sectors, Nucl. Phys. B925 (2017) 1 [1705.05314].

[41] D. de Florian, I. Fabre and J. Mazzitelli, Higgs boson pair production at NNLO in QCD including dimension 6 operators, JHEP 10 (2017) 215 [1704.05700].

[42] G. Buchalla, M. Capozi, A. Celis, G. Heinrich and L. Scyboz, Higgs boson pair production in non-linear Effective Field Theory with full $m_{t}$-dependence at NLO QCD, JHEP 09 (2018) 057 [1806.05162].

[43] M. Capozi and G. Heinrich, Exploring anomalous couplings in Higgs boson pair production through shape analysis, 1908.08923.

[44] C.-R. Chen and I. Low, Double take on new physics in double Higgs boson production, Phys. Rev. D90 (2014) 013018 [1405. 7040$].$

[45] A. Azatov, R. Contino, G. Panico and M. Son, Effective field theory analysis of double Higgs boson production via gluon fusion, Phys. Rev. D92 (2015) 035001 [1502.00539]. 
[46] S. Dawson, A. Ismail and I. Low, What's in the loop? The anatomy of double Higgs production, Phys. Rev. D91 (2015) 115008 [1504.05596].

[47] A. Carvalho, M. Dall'Osso, T. Dorigo, F. Goertz, C. A. Gottardo and M. Tosi, Higgs Pair Production: Choosing Benchmarks With Cluster Analysis, JHEP 04 (2016) 126 [1507 . 022 4 5].

[48] A. Carvalho, M. Dall'Osso, P. De Castro Manzano, T. Dorigo, F. Goertz, M. Gouzevich et al., Analytical parametrization and shape classification of anomalous $H H$ production in the EFT approach, 1608.06578.

[49] A. Carvalho, F. Goertz, K. Mimasu, M. Gouzevitch and A. Aggarwal, On the reinterpretation of non-resonant searches for Higgs boson pairs, 1710.08261.

[50] https://www.scikit-learn.org.

[51] G. Buchalla, O. Cata, A. Celis and C. Krause, Note on Anomalous Higgs-Boson Couplings in Effective Field Theory, Phys. Lett. B750 (2015) 298 [1504.01707].

[52] https://keras.io.

[53] https://www.tensorflow.org.

[54] D. P. Kingma and J. Ba, Adam: A Method for Stochastic Optimization, 1412.6980.

[55] J. Butterworth et al., PDF4LHC recommendations for LHC Run II, J. Phys. G43 (2016) 023001 [1510.03865]. 